\title{
A Collaborative Approach to Reduce the Spread of COVID-19 in a Large Urban Hospital in South Florida
}

\author{
By: David M. Schneider, BSN, RN, CMSRN; Roberto L. Roman Laporte, DNP, RN, CMSRN, EBP-C
}

\begin{abstract}
The novel Coronavirus has proven challenging for many healthcare organizations. Hospitals are pressed to secure sufficient personal protective equipment to ensure the safety of their healthcare personnel and various strategies have been implemented to stop the spread of the virus. There are still many unknowns about COVID-19 and hospitals are rapidly adapting to new information while trying to maintain the safety and wellbeing of healthcare personnel. Baptist Hospital of Miami developed a collaborative data driven plan to monitor, disseminate vital information, and implement various strategies to protect employees and patients during the pandemic.
\end{abstract}

Keywords: Contract tracing, COVID-19, acute care, Coronavirus

\section{INTRODUCTION}

December 31, 2019, China reports an atypical presentation of pneumonia flooding their healthcare system (World Health Organization, 2020). This atypical pneumonia, soon to be identified as COVID-19, quickly swept across the globe. Anyone that was exposed to a positive COVID-19 case or traveled from a high-risk area was recommended to self-quarantine for fourteen days. In the early days of COVID-19, a pandemic seemed like an impossibility in the United States; the last severe pandemic was over 100 years ago (Centers for Disease Control and Prevention, 2019). The US cautiously waited to see how to respond to the looming pandemic. Hot spots were quickly growing from coast to coast across the US. The fear of over burdening the US healthcare system was becoming a reality (Kaste, 2020). New York's healthcare system quickly surged past capacity. Soon after, Miami Dade County would be the hardest hit county in the state of Florida with over 17,000 confirmed COVID-19 cases (Florida Health, 2020). Hospitals across the US were challenged to develop surge plans to treat COVID-19 cases.

In Miami-Dade County, Baptist Hospital of Miami (Baptist Hospital), the largest hospital within Baptist Health of South Florida had to prepare for the unknown. Baptist Hospital is no stranger to preparing for disaster - the annual hurricane season tests even the most seasoned emergency response crews. Baptist Hospital had to address a central issue: how to protect its employees and patients from spreading the virus to each other? COVID-19 is believed to have a very high risk for intrahospital spread among healthcare personnel with a R0 factor of 2.0 to 2.5 (Fischer, 2020). If an employee contracted the virus, he or she had to quarantine for fourteen days. In addition to the recommended Centers for Disease Control and Prevention (CDC) guidelines for healthcare personnel screening, the answer to solving this was far more than personal protective equipment (PPE). Baptist Hospital established a systematic approach of contact tracing to identify in-house exposures and link them to any possible points of contact to other individuals. If Baptist Hospital, was to weather the storm, it would need all staff healthy and ready to fight, not sidelined in quarantine.

\section{COVID-19 INITIATIVE}

The first case of COVID-19 in Miami was reported in early March, 2020. Baptist Hospital mobilized a plan soon after and a collaborative approach was developed between all levels of employees within the organization. The plan was a four-pronged approach to ensure the safety of healthcare personnel and patients: 1) frontline 
leaders reported exposures in real time to a central exposure team. The Performance Improvement (PI) department was the designated team, available around the clock; 2) PI reviewed and communicated the cases to the Infection Prevention and Control (IPC) team using a standard communication tool; 3) IPC reviewed any COVID-19 related cases, often collaborating with infectious disease physicians, and provided an exposure risk determination (low, medium, or high risk). Once a decision was made, the leader of the unit would be notified and, if warranted, the employee would be sent to self-quarantine; and 4) the Occupational Health Office followed up with team members throughout the quarantine, facilitated further testing, and coordinated the return-to-work process.

Baptist Hospital's IPC team quickly became a symbol of hope and safety to frontline workers. Members of the IPC team would frequently round on the units evaluating how the teams were managing PPE, ensuring containment of the virus, and offering real time education and facilitating question and answer sessions with staff on anything related to PPE and coronavirus. Early execution of these rounds provided valuable insight on the organization's strengths and weaknesses in virus containment and allowed the organization to shift fluidly to new procedures and products to best protect the healthcare personnel and patients.

In anticipation of the inevitable surge, real time data became one of the most valuable tools in the continued preparedness for the fight against the COVID-19. The PI team launched an in-depth and reliable data source on which Baptist Hospital could rely for up-to-date information on a myriad of COVID-19 data points including key points such as: number of positive and negative cases tested in house, number of patients pending results, number of patients extubated, discharged home, or expired. PI regularly communicated the data to all levels within the hospital to ensure consistency and to determine if extra measures were necessary to combat this evolving situation and plan accordingly. Real time data, effective change, and a determined healthcare team allowed Baptist Hospital to have a very successful response to the pandemic. As the percent of new cases trended down, reported data shifted towards a focus on discharges and extubations. As the COVID-19 recovery phase progresses and in awareness of a potential second wave, Baptist Hospital of Miami continues to monitor this data regularly.

In addition to contact tracing and data monitoring, Baptist Hospital utilized several other methodologies to stay ahead of the impending COVID-19 surge. Early on in the pandemic, Baptist Hospital, relocated patients and created dedicated units to treat patients diagnosed with COVID-19 in an attempt to limit exposure and cross-contamination. In addition to dedicated units, COVID-19 screening checkpoints were strategically set up in key entrances throughout the hospital to control access. Temperature screenings, mask allotments, and badge identification were mandated at each check-in point. During heightened security, visitor access, including students, was restricted. To aid in communication and support of patients, the hospital utilized iPads as a tele-communication tool for patients and families. The iPads, with video communication capabilities, safely provided family members a chance to be a part of the healthcare process during a period when visitors are not permitted to visit patients suspected or diagnosed with COVID-19. More importantly, the iPads gave the patient an opportunity to spend time with family.

Although the novel Coronavirus continues to be a threat to public safety, adherence to social distancing, proper use of masks/face coverings and meticulous hand hygiene can help mitigate the negative effects of the disease and ultimately slow the spread of the virus. Even with the best efforts, the virus has managed to leave a destructive path, impacting the lives of millions around the world. Baptist Hospital, armed with a dedicated healthcare team and an ability to develop creative data driven solutions to complex problems with fluidity, is prepared to continue fighting the virus to provide the high quality of care the South Florida community deserves and expects. 


\section{COVID-19 Special Edition}

\section{DECLARATION OF INTEREST}

The authors report no conflicts of interest. The authors alone are responsible for the content and writing of the paper.

\section{AUTHORS}

David M. Schneider, BSN, RN, CMSRN, Performance Improvement Nurse, Baptist Hospital of Miami, FL, US. Correspondence regarding this paper can be directed at:

MrDavidSchneider@gmail.com

\section{Roberto L. Roman Laporte, DNP, RN,}

CMSRN, EBP-C, Nurse Scientist, Baptist

Health South Florida, Miami, FL, US. Corre-

spondence regarding this pa-

per can be directed at:

RobertRL@baptisthealth.net

\section{REFERENCES}

Centers for Disease Control and Prevention. (2019). 1918 pandemic (H1N1 virus). https://www.cdc.gov/flu/pandemicresources/1918-pandemic-hln1.html

Fisher, M. (2020). R0 the messy metric that shape may soon shape our lives, explained. The New York Times. https://www.nytimes.com/2020/04/23/world/ europe/coronavirus-R0-explainer.html

Florida Health. (2020). Florida COVID-19 response. https://floridahealthcovid19.gov/

Kaste, M. (2020). U.S. hospitals prepare for a COVID-19 wave. https:// www.npr.org/2020/03/06/812967454/u-shospitals-prepare-for-a-COVID-19-wave

World Health Organization. (2020). Pneumonia of unknown cause - China. https://www.who.int/csr/don/05-january-2020 -pneumonia-of-unkown-cause-china/en/ 